\title{
DYNAMICS AND CONTROL OF THE GYROSCOPIC HEAD USED FOR THE LASER ILLUMINATION OF A GROUND TARGET FROM THE QUADCOPTER DECK
}

\author{
Zbigniew Koruba, Izabela Krzysztofik* \\ Faculty of Mechatronics and Mechanical Engineering, Kielce University of Technology, Kielce, Poland \\ *E-mail of corresponding author: pssik@tu.kielce.pl
}

\begin{abstract}
Resume
In the paper authors investigate dynamics of a controlled quadcopter in terms of the possibility of its use for detection, observation, tracking and laser illuminating of both stationary and moving ground targets in the conditions of impact of random and kinematic excitations. The drone is equipped with a scanning and tracking Gyroscopic Head (GH) coupled with a laser target indicator. The drone is affected by random disturbances in the form of wind gusts or explosions of missiles. Kinematic excitations, such as drone maneuvers and vibrations from engines, act on the GH. This paper focuses mainly on control and stabilization of the gyroscopic head placed on the drone during the search, tracking and simultaneous laser illuminating of the target.
\end{abstract}

Available online: https://doi.org/10.26552/com.C.2021.2.B158-B164

\section{Article info}

Received 17 September 2020

Accepted 29 October 2020

Online 11 March 2021

\section{Keywords:}

control and dynamics of quadcopter, scanning and tracking by gyroscopic head,

modeling and guidance
ISSN 1335-4205 (print version)

ISSN 2585-7878 (online version)

\section{Introduction}

In recent years, four-rotor unmanned aerial vehicles (quadcopters) have become a very popular unmanned platform that is still in the stage of intensive research and finds a variety of applications. Currently, they are used, among others, for shooting collective events, monitoring communication infrastructure, supporting rescue operations, monitoring the state of air pollution. The paper proposes the use of this type of QuadCopter Unmanned Aerial Vehicle (QCUAV) for the laser pointing of ground targets, both stationary and mobile, using the scanning and tracking gyroscopic head [1-2]. In addition, a mathematical model was developed for controlling the movement of the gyroscopic scanning and tracking head as well as the mathematical model of the drone's dynamics [3-5].

It should be emphasized that the advantage of the considered QCUAV is its ability to stay in hover for a certain period of time. The system of laser pointing of a target (SLPT) can thus act independent from drone's movement and therefore stably determines the target observation line (T-LOS) regardless of disturbances acting on the drone itself. While searching for a target, the GH is put into a programmed motion that allows scanning the ground surface [6]. The infrared sensor and the laser target pointer are attached to the GH axis. At the moment of receiving a thermal signal from the target, the drone goes to hover and GH begins to illuminate it with laser pulses, so that target can become the object of attack [7-8].

To ensure the high precision in maintaining the T-LOS in a given position, an optimal controller was designed for the GH. For this reason, despite many control methods used for the unmanned aerial vehicle, known in the literature [9-11] a simple PID controller was used to control the flight of the QCUAV, since the drone movement in space does not have to be precisely determined [12-16]. Simplified operation diagram of the system of detection, tracking and laser pointing of a target from the QCUAV deck is shown in Figure 1.

\section{Mathematical model of dynamics of the QCUAV and controlled gyroscopic head}

The executive unit in the scanning and tracking head, mentioned above, is a controlled gyroscope system. The general view of the gyroscope system with forces and moments of forces is shown in Figure 2.

Mathematical model of the controlled gyroscope system dynamics is presented in the following form [17]:

$$
\begin{aligned}
& J_{y 1} \frac{d \omega_{g y 1}}{d t}+\left(J_{y 2}+J_{y 3}\right) \frac{d}{d t}\left(\omega_{g y 2} \cos \theta_{g}\right)-J_{z 2} \times \\
& \times \frac{d}{d t}\left(\omega_{g z 2} \sin \theta_{g}\right)-J_{z 3} \frac{d}{d t}\left(\omega_{g z 3} \sin \theta_{g}\right)+m_{g} l_{g} \times \\
& \times \frac{d}{d t}\left[V_{g x 2}\left(1+\cos \theta_{g}\right)\right]+\left(J_{x 1}+J_{z 1}\right) \omega_{g x 1} \omega_{g z 1}+ \\
& +\left(J_{x 2}+J_{x 3}\right) \omega_{g x 2} \omega_{g z 1}-\left(J_{y 2}+J_{y 3}\right) \omega_{g y 2} \omega_{g x 1} \sin \theta_{g}-
\end{aligned}
$$




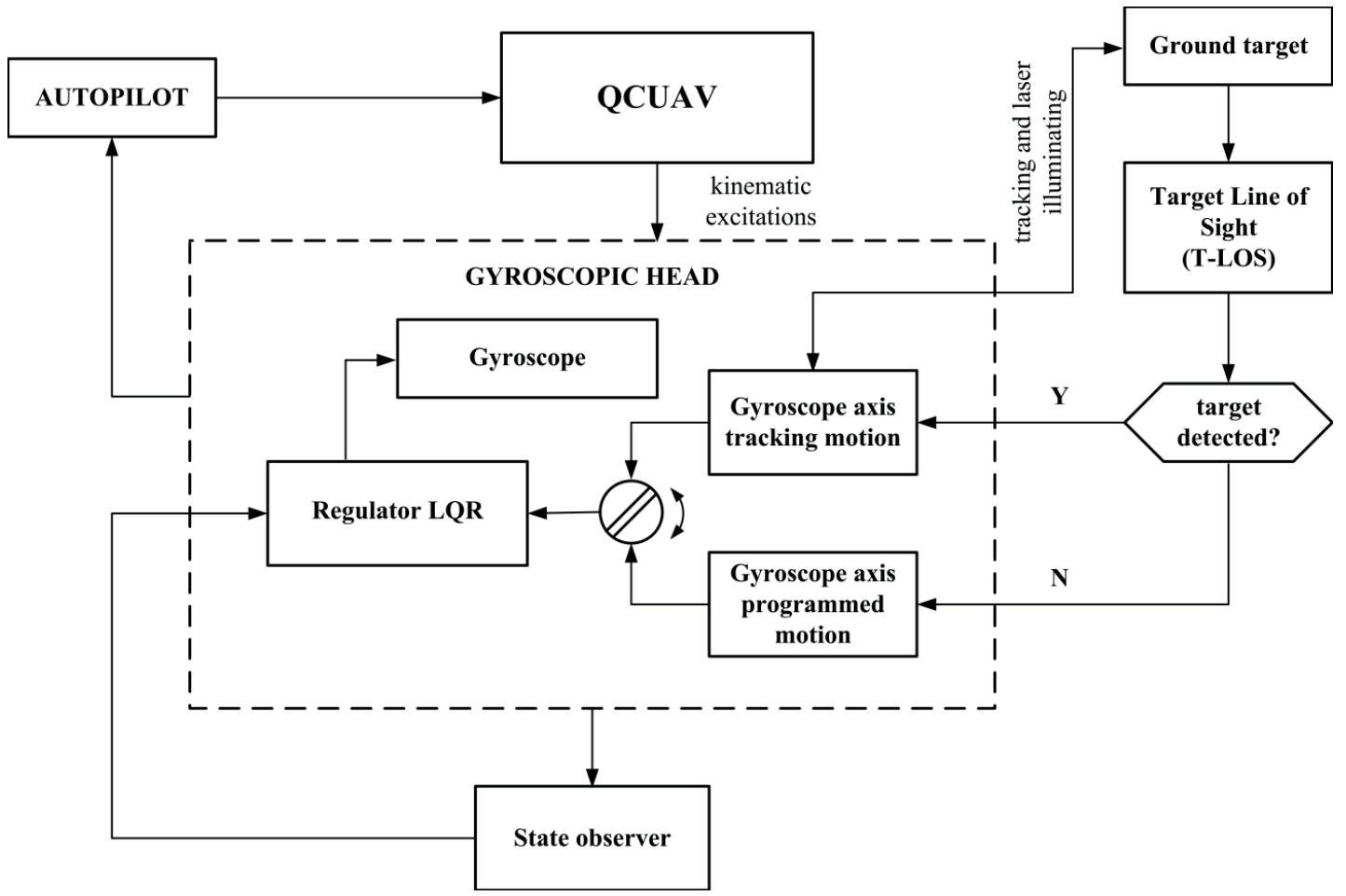

Figure 1 Simplified operation diagram of the system of detection, tracking and laser pointing of a target from the QCUAV deck

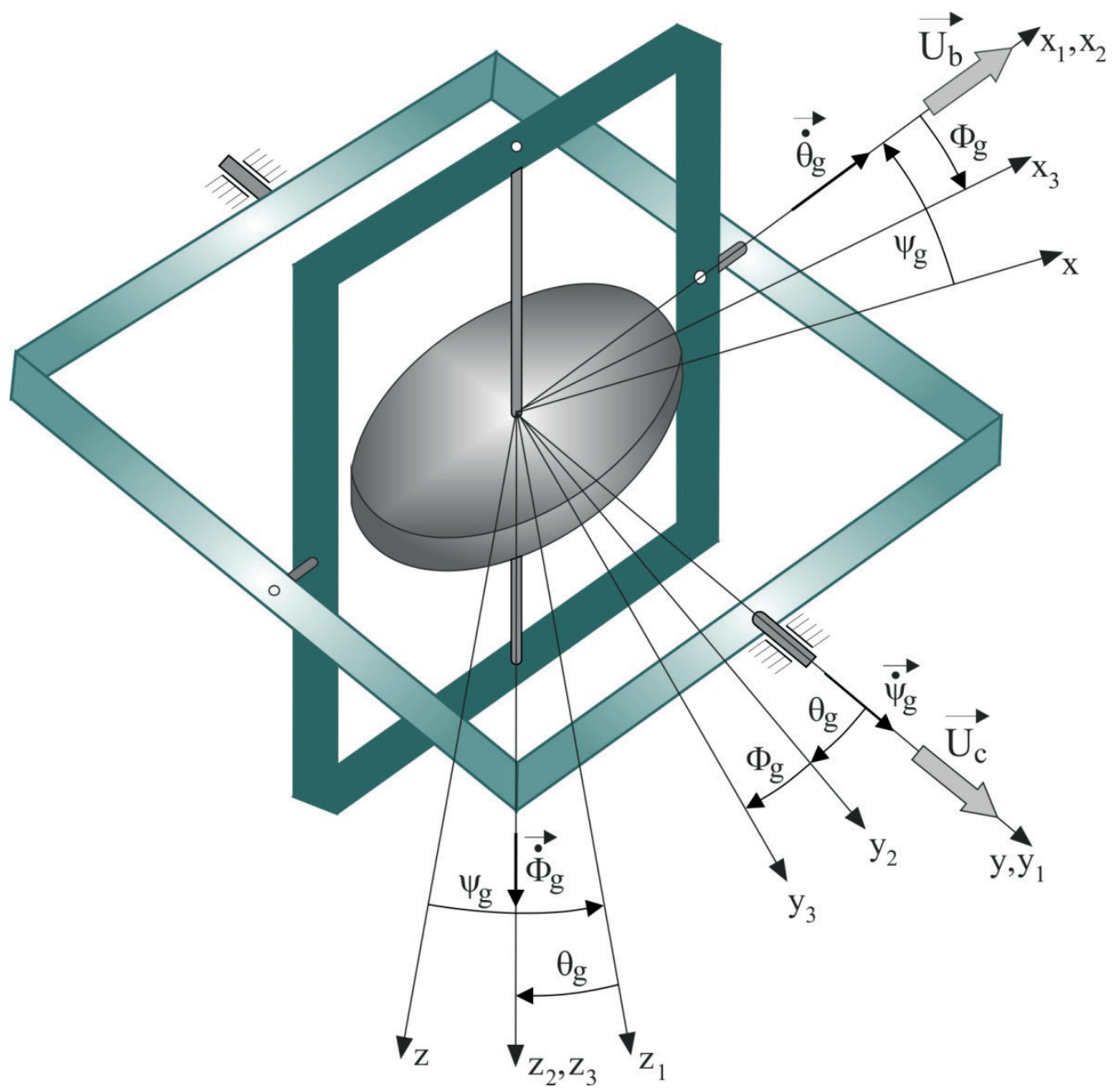

Figure 2 General view of the gyroscope system 


$$
\begin{aligned}
& -\left(J_{z 2} \omega_{g z 2}+J_{z 3} \omega_{g z 3}\right) \omega_{g x 1} \cos \theta_{g}-m_{g} V_{g z 2}\left[-V_{g z 1}+\right. \\
& \left.+\omega_{g x 1} l_{g} \sin \theta_{g}\right]-m_{g} V_{g y 2}\left[V_{g x 1} \sin \theta_{g}+\omega_{g z 1} l_{g} \times\right. \\
& \left.\left(1+\cos \theta_{g}\right)\right]-m_{g} V_{g z 2}\left[V_{g x 1} \cos \theta_{g}-\omega_{g z 1} l_{g} \sin \theta_{g}\right]= \\
& =U_{c}-U_{c f},
\end{aligned}
$$

$$
\begin{aligned}
& \left(J_{x 2}+J_{x 3}\right) \frac{d \omega_{g x 2}}{d t}-m_{g} l_{g} \frac{d V_{g y 2}}{d t}-\left(J_{y 2}+J_{y 3}-J_{z 2}\right) \times \\
& \times \omega_{g y 2} \omega_{g z 2}-J_{z 3} \omega_{g z 3} \omega_{g y 2}-m_{g} l_{g} V_{g x 2} \omega_{g z 2}-m_{g} V_{g y 2} \times \\
& \times V_{g z 2}-m_{g} V_{g z 2}\left[-V_{g z 1} \sin \theta_{g}-V_{g y 1} \cos \theta_{g}+\omega_{g x 1} \times\right. \\
& \left.\times l_{g} \cos \theta_{g}\right]=U_{b}-U_{b f},
\end{aligned}
$$

where:

$$
\begin{aligned}
& \omega_{g x 1}=p_{v} \cos \phi_{g}-r_{v} \sin \phi_{g} ; \omega_{g y 1}=\dot{\phi}_{g}+q_{v} ; \\
& \omega_{g z 1}=p_{v} \sin \phi_{g}+r_{v} \cos \phi_{g} \\
& \omega_{g x_{2}}=\omega_{g x_{1}}+\dot{\theta}_{g} \text {; } \\
& \omega_{g y_{2}}=\omega_{g z_{1}} \sin \theta_{g}+\omega_{g y_{1}} \cos \theta g \text {; } \\
& \omega_{g z 2}=\omega_{g z 1} \cos \theta_{g}+\omega_{g y 1} \sin \theta g \\
& \omega_{g z 3}=\omega_{g z 2}+n_{g} \text {; } \\
& V_{g x 1}=u_{v} \cos \phi_{g}-w_{v} \sin \phi_{g} ; V_{g z 1}=v_{v} ; \\
& V_{g z 1}=u_{v} \sin \phi_{g}-w_{v} \cos \phi_{g} \text {; } \\
& V_{g x 2}=V_{g x 1}+l_{g}\left(\omega_{g y 1}+\omega_{g y 2}\right) \text {; } \\
& V_{g y 2}=V_{g z 1} \sin \theta_{g}+V_{g y 1} \cos \theta_{g}-l_{g}\left(\omega_{g x 1} \cos \theta_{g}+\omega_{g x 2}\right) \text {; } \\
& V_{g z 2}=V_{g z 1} \cos \theta_{g}-V_{g y_{1}} \sin \theta_{g}+l_{g} \omega_{g x_{1}} \sin \theta_{g} ; \\
& \text { and }
\end{aligned}
$$$$
\phi_{g}, \theta_{g}, \Phi_{g} \text { - angles determining the rotor position in space; }
$$$$
p_{v}, q_{v}, r_{v} \text { - components of the quadcopter angular speed; }
$$$$
u_{v}, v_{v}, w_{v} \text { - components of the quadcopter linear speed; }
$$$$
J_{x_{1}}, J_{y_{1}}, J_{z 1} \text { - moments of inertia of the external frame; }
$$$$
J_{x_{2}}, J_{y_{2}}, J_{z_{2}} \text { - moments of inertia of the internal frame; }
$$$$
J_{x 3}, J_{y_{3}}, J_{z 3} \text { - moments of inertia of the rotor; }
$$$$
n_{g} \text { - rotation speed of the rotor; }
$$$$
m_{g} \text { - mass of the internal frame and rotor; }
$$$$
l_{g} \text { - distance from the gyroscope mass centre to the }
$$$$
\text { gyroscope centre of rotation; }
$$

$\eta_{b}, \eta_{c}$ - friction coefficients in the suspension bearings;

$U_{b}, U_{c}$ - gyroscope control moments;

$U_{b f}, U_{c f}$ - moments of friction forces in the bearings of the internal and external frame respectively, determined by relationships:

for viscous $U_{b f}=\eta_{b} \dot{\theta}_{g} ; U_{c f}=\eta_{c} \dot{\phi}_{g}$ and

for dry friction $U_{b f}=\frac{1}{2} d_{b f} T_{b f} ; U_{c f}=\frac{1}{2} d_{c} T_{c f}$

with: $T_{b f}=\mu_{b} N_{b} \operatorname{sign}\left(\dot{\theta}_{g}\right) ; T_{c f}=\mu_{c} N_{c} \operatorname{sign}\left(\dot{\phi}_{g}\right)$

$\eta_{b}, \eta_{c}, \mu_{b}, \mu_{c}$ - friction coefficients in the suspension bearings;

$N_{b}, N_{c}$ - normal reactions in the frame bearings;

$d_{b}, d_{c}$ - diameters of bearing pivots.

Occurrence of friction in the suspension bearings causes formation of moments acting on gyroscope, depending on the angular velocity of the vehicle on which the gyroscope is located. Assuming that the moments of friction forces are of the viscous type, one has:

$$
U_{b f}=\eta_{b} \dot{\phi}_{v} ; U_{c f}=\eta_{c} \dot{\theta}_{v}
$$

Out of numerous gyro control methods [17], the optimum LQR method has been adopted in this paper [1820]. The control law $\mathbf{u}_{g}$ for GH was determined using the linear-quadratic optimization method with the function in the form of:

$$
I=\int_{0}^{\infty}\left(\mathbf{x}_{\mathrm{g}}^{\mathrm{T}} \mathbf{Q} \mathbf{x}_{\mathrm{g}}+\mathbf{u}_{\mathrm{g}}^{\mathrm{T}} \mathbf{R} \mathbf{u}_{\mathrm{g}}\right) d t .
$$

This law is presented by equation:

$\mathbf{u}_{\mathrm{g}}=-\mathbf{K}_{\mathrm{g}}\left(\mathbf{x}_{\mathrm{g}}-\mathbf{x}_{\mathrm{gz}}\right)$,

where $\mathbf{x}_{\mathrm{g}}$ is a vector of actual state variables determining the position of gyroscope system axis in space and $\mathbf{x}_{\mathrm{g}_{\mathrm{z}}}$ is a set (desired) state variables.

The $\mathbf{K}_{\mathbf{g}}$ matrix of gains is determined using the Matlab function [21-22]:

$\mathbf{K}_{\mathrm{g}}=\operatorname{lqr}(\mathbf{J}, \mathbf{B}, \mathbf{Q}, \mathbf{R})$,

where $\mathbf{J}$, matrix constituting the argument of $l q r$ function, is a Jacobian of the gyroscope system.

To determine components of Jacobian J, the motion Equations (1)-(2) were simplified to the following form, respectively:

$$
\begin{aligned}
& J_{g k} \ddot{\phi}_{g}+J_{g k} \dot{\phi}_{g} \dot{\theta}_{g} \sin 2 \theta_{g}+J_{g o} n_{g} \dot{\theta}_{g} \cos \theta_{g}= \\
& =U_{c}-U_{c f}, \\
& J_{g k} \ddot{\theta}_{g}-0.5 J_{g k}\left(\dot{\phi}_{g}\right)^{2} \sin 2 \theta_{g}-J_{g o} n_{g} \dot{\phi}_{g} \cos \theta_{g}= \\
& =U_{b}-U_{b f},
\end{aligned}
$$

where:

$J_{g k}=J_{x 3}=J_{y 3}$ and $J_{g o}=J_{z 3}$.

Then, components of the J matrix are determined as follow:

$J_{11}=0, J_{12}=1, J_{13}=0, J_{14}=0$;

$J_{21}=\left(-J_{g k} \dot{\phi}_{g}^{2} \cos 2 \theta_{g}+J_{g o} n_{g} \dot{\phi}_{g} \sin \theta_{g}\right) / J_{g k}$;

$J_{22}=-\frac{n_{b}}{J_{g k}}, J_{23}=0, J_{24}=\left(\begin{array}{l}-J_{g k} \dot{\phi}_{g} \sin 2 \theta_{g}- \\ -J_{g o} n_{g} \cos \theta_{g}\end{array}\right) / J_{g k} ;$

$J_{31}=0, J_{32}=0, J_{33}=0, J_{34}=1$;

$J_{41}=2 \sin \theta_{g}\left(\begin{array}{l}-\eta_{c} \dot{\phi}_{g}+J_{g k} \dot{\vartheta}_{g} \dot{\phi}_{g} \sin 2 \vartheta_{g}+ \\ +J_{g o} n_{g} \dot{\vartheta}_{g} \cos \theta_{g}\end{array}\right) / J_{g k} \cos ^{3} \theta_{g}$

$+\left(2 J_{g k} \dot{\theta}_{g} \dot{\phi}_{g} \cos 2 \theta_{g}-J_{g o} n_{g} \dot{\theta}_{g} \sin \vartheta_{g}\right) / J_{g k} \cos ^{2} \theta_{g} ;$

$J_{41}=\left(J_{g k} \dot{\phi}_{g} \sin 2 \theta_{g}+J_{g o} n_{g} \cos \vartheta_{g}\right) / J_{g k} \cos ^{2} \theta_{g} ;$

$J_{43}=0, J_{44}=\left(-\eta_{c}+J_{g k} \dot{\theta}_{g} \sin 2 \vartheta_{g}\right) / J_{g k} \cos ^{2} \theta_{g}$.

If the state observer is used, instead of the $\mathbf{x}_{\mathrm{g}}$ state, the regulator is entered by an estimate $\hat{\mathrm{x}}_{\mathrm{g}}$ from the observer, i.e. the control will take the form of

$\mathbf{u}_{\mathrm{g}}(\mathrm{t})=-\mathbf{K}_{\mathrm{g}}(\mathrm{t}) \hat{\mathbf{x}}_{\mathrm{g}}(\mathrm{t})$.

The QCUAV configuration with rotational speeds, forces and moments generated by the four motors is presented in Figure 3.

Using the Euler-Lagrange formalism, the following mathematical model of the quadcopter dynamics was derived: 


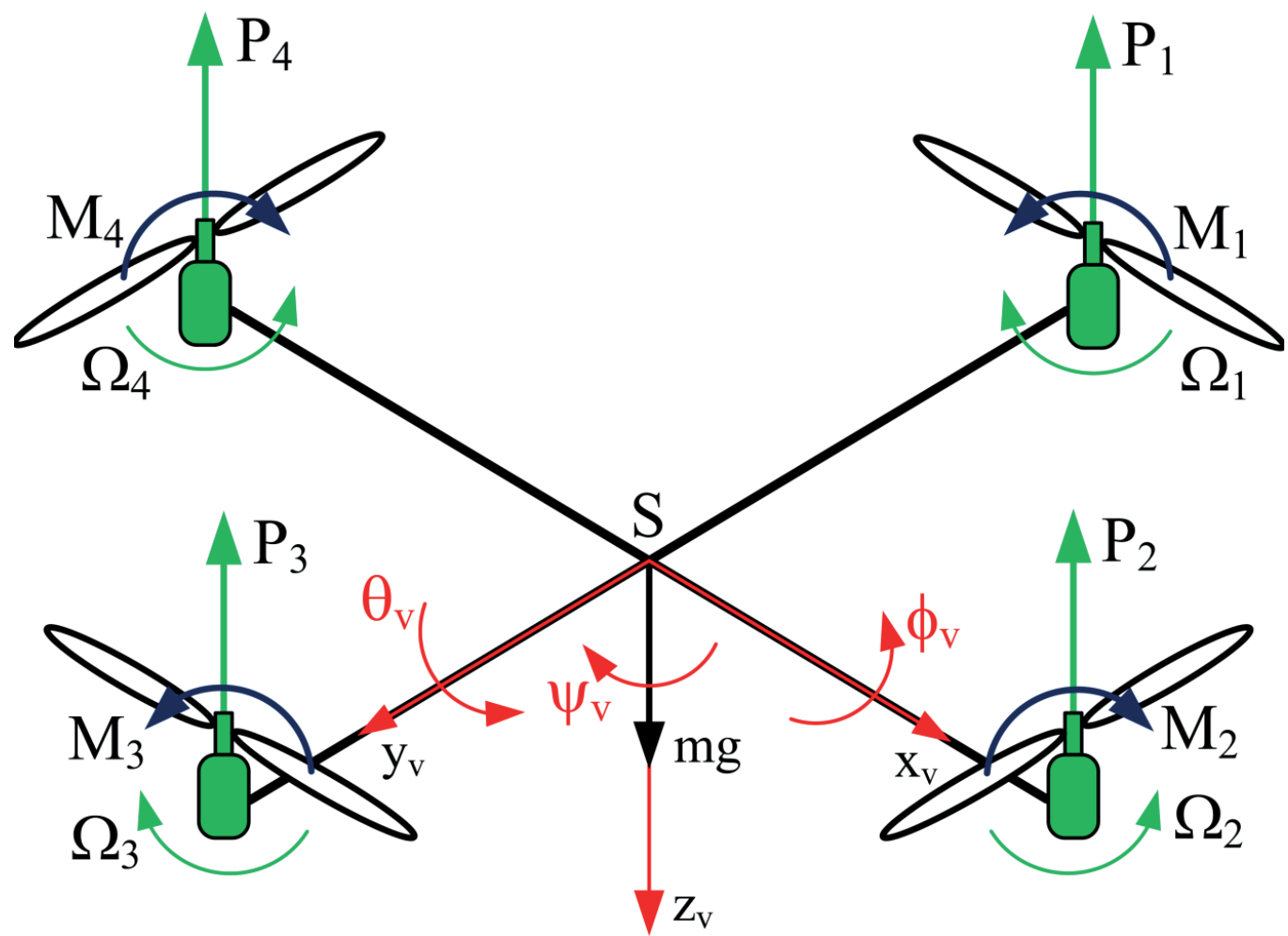

Figure 3 Angles of the quadcopter position

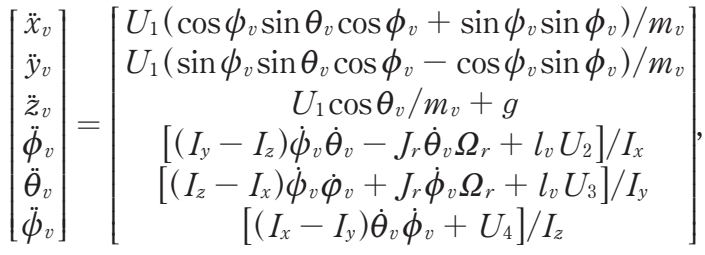

with

$\Omega_{r}=\Omega_{1}+\Omega_{3}-\Omega_{2}-\Omega_{4}$;

$\Omega_{1}, \Omega_{3}, \Omega_{2}, \Omega_{4}$ - rotational speeds of the individual motors,

$l_{v}$ - arm's length,

$m_{v}$ - drone's mass,

$I_{x}, I_{y}, I_{z}$ - moments of inertia of the QCUAV,

$J_{r}$ - moment of inertia of the rotor and

$\mathrm{U}_{1}, \mathrm{U}_{2}, \mathrm{U}_{3}, \mathrm{U}_{4}$ - controls.

The control vector $\mathbf{U}$ is determined by the following formula:

$\left[\begin{array}{c}U_{1} \\ U_{2} \\ U_{3} \\ U_{4}\end{array}\right]=\left[\begin{array}{c}-b\left(\Omega_{1}^{2}+\Omega_{2}^{2}+\Omega_{3}^{2}+\Omega_{4}^{2}\right) \\ b\left(\Omega_{1}^{2}-\Omega_{3}^{2}\right) \\ b\left(\Omega_{2}^{2}-\Omega_{4}^{2}\right) \\ d\left(\Omega_{2}^{2}+\Omega_{4}^{2}-\Omega_{1}^{2}-\Omega_{3}^{2}\right)\end{array}\right]$

where $b$ is the thrust coefficient and $d$ is the drag coefficient.

\section{$3 \quad$ Results and conlusions}

In order to verify the correct operation of the gyro head during the laser illuminating the ground target from the deck of drone, appropriate simulation tests were carried out. The drone movement from a given starting point in space to a point on a fixed height $H_{d o}=20 \mathrm{~m}$ - exactly above and at a fixed distance from the target, moving on the surface of the earth, was considered. The drone's GH tracks the moving target and at the same time illuminates it with a laser. This is shown in the Figures 4-12.

The tests were conducted in the Matlab/Simulink environment using the ode 45 integration procedure for the following parameters:

- gyro system parameters

$J_{x 1}=2.5 \cdot 10^{-5} \mathrm{kgm}^{2} ; J_{y_{1}}=J_{x 1} ; I_{z 1}=J_{x 1} ;$

$J_{x 2}=5 \cdot 10^{-5} \mathrm{kgm}^{2} ; J_{y 2}=J_{x 2} ; I_{z 2}=J_{x 2} ;$

$J_{x 3}=2.5 \cdot 10^{-4} \mathrm{kgm}^{2} ; J_{y_{3}}=J_{x 3} ; I_{z 3}=5 \cdot 10^{-4} \mathrm{kgm}^{2}$;

$n_{g}=600 \mathrm{rad} / s ; \eta_{b}=0.01 \mathrm{Nms} ; \eta_{c}=0.01 \mathrm{Nms} ;$

$m_{g}=0.24 \mathrm{~kg} ; l_{g}=0.001 \mathrm{~m}$;

optimal LQR gain matrix for the gyro system

$K_{g}=\left[\begin{array}{cccc}9.5114 & 0.3709 & 41.9258 & 0.1695 \\ -2.0134 & 0.0004 & 0.4585 & 0.0031\end{array}\right]$

- quadcopter parameters

$m_{v}=0.65 \mathrm{~kg} ; l_{v}=0.23 \mathrm{~m} ; b=3.13 \cdot 10^{-5} \mathrm{Nms}^{2}$;

$d=7.5 \cdot 10^{-7} \mathrm{Nms}^{2} ; J_{r}=6.0 \cdot 10^{-5} \mathrm{kgm}^{2}$;

$\Omega_{\max }=500 \mathrm{rad} / \mathrm{s} ; I_{x}=7.5 \cdot 10^{-3} \mathrm{kgm}^{2}$;

$I_{y}=7.5 \cdot 10^{-3} \mathrm{kgm}^{2} ; I_{z}=1.3 \cdot 10^{-2} \mathrm{kgm}^{2} ;$

the values of the PID regulator coefficients for the drone

$k_{p 1}=11.8 ; k_{d 1}=10.8 ; k_{i 1}=0.9$;

$k_{p 2}=0.65 ; k_{d 2}=0.46$;

$k_{p 3}=0.45 ; k_{d 3}=0.36$;

$k_{p 4}=0.12 ; k_{d 4}=0.12$;

$k_{p x}=8.8 ; k_{d x}=8.9$;

$k_{p y}=8.8 ; k_{d y}=8.9$.

The graphs show the movement of the gyroscope axis, having the task at keeping the line of sight from 


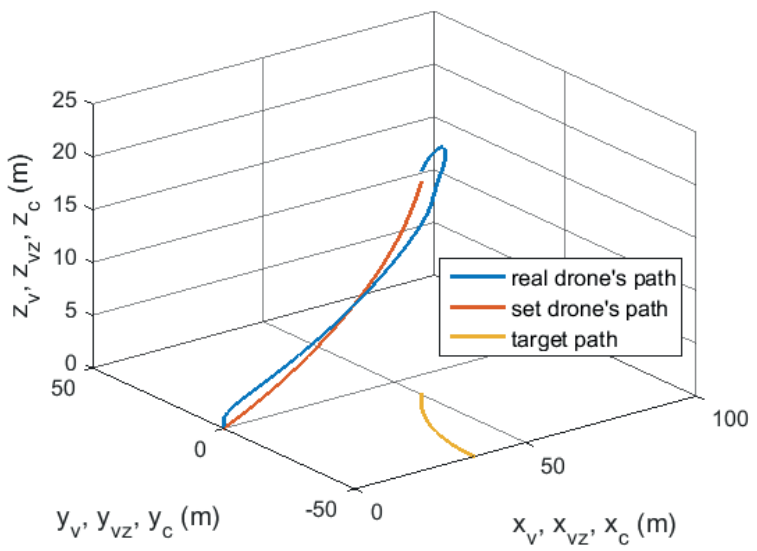

Figure 4 Drone's flight path and target trajectory

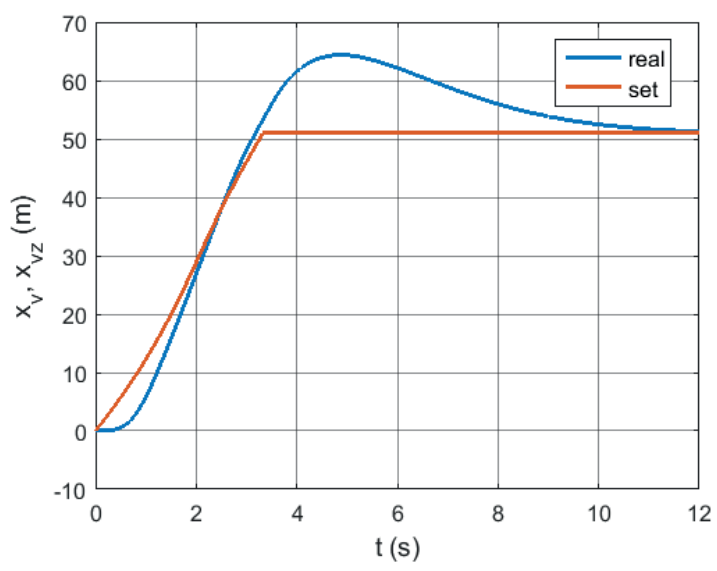

Figure 5 Changes of the state variables $x_{v}$ and $x_{v z}-$ real and set - as a function of time

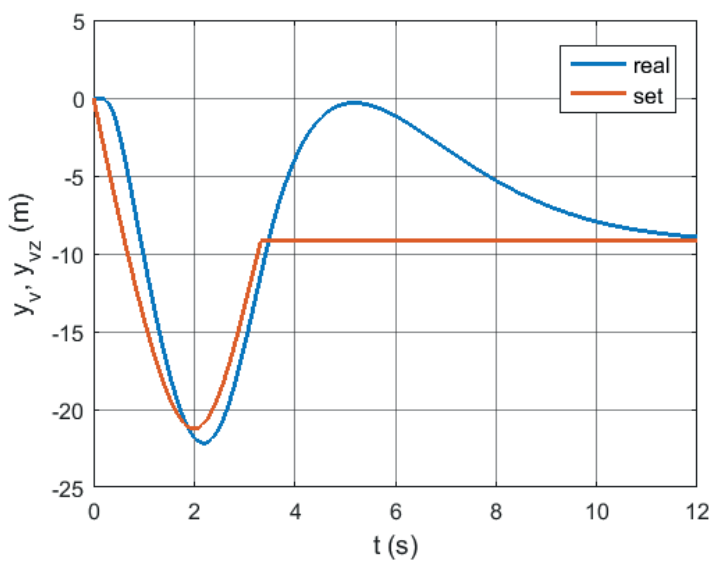

Figure 5 Changes of the state variables $y_{v}$ and $y_{v z}-$ real and set - as a function of time

the deck of the drone from the moment of gaining the height to the passage and maintaining the hover. At the starting moment of the drone, the initial conditions of the gyroscope axis motion are unknown. For this reason, a series compensator was used (the state observer with the LQR controller). In the initial phase of tracking, i.e. for a period of about 1 second, the large dynamic effects are

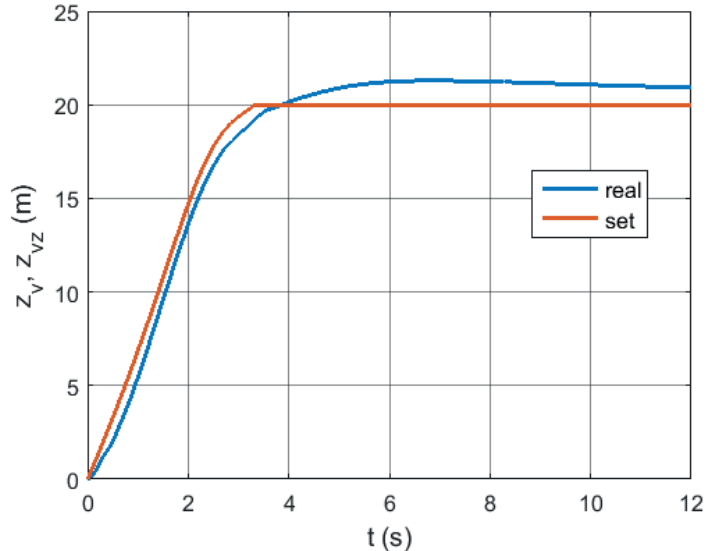

Figure 6 Changes of the state variables $z_{v}$ and $z_{v z}$-real and set - as a function of time

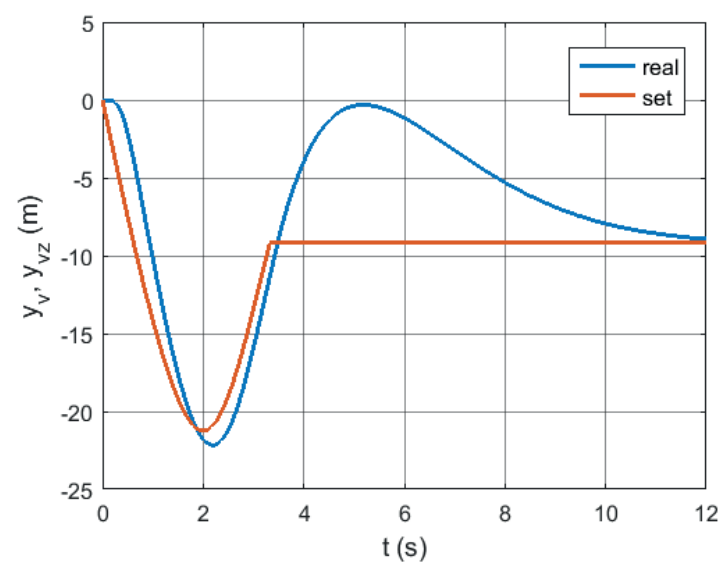

Figure 5 Changes of the state variables $y_{v}$ and $y_{v z}$ - real and set - as a function of time

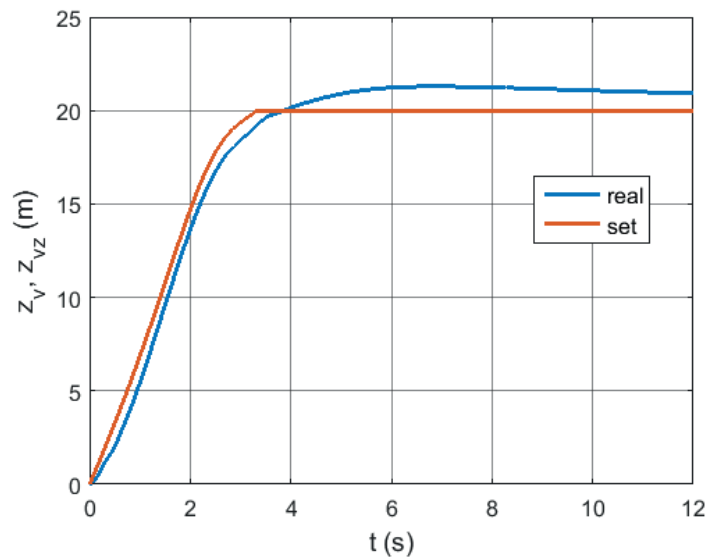

Figure 6 Changes of the state variables $z_{v}$ and $z_{v z}$ - real and set - as a function of time

visible, after which the compensator follows the target with sufficient accuracy. However, it should be noted, that the transitional period also appears when the drone is moved to hover and lasts only about $0.5 \mathrm{~s}$. It was also assumed that during the reaching heights and hover, the wind is blowing on the drone. These gusts significantly disturb the drone flight trajectory, however, they do not have much 


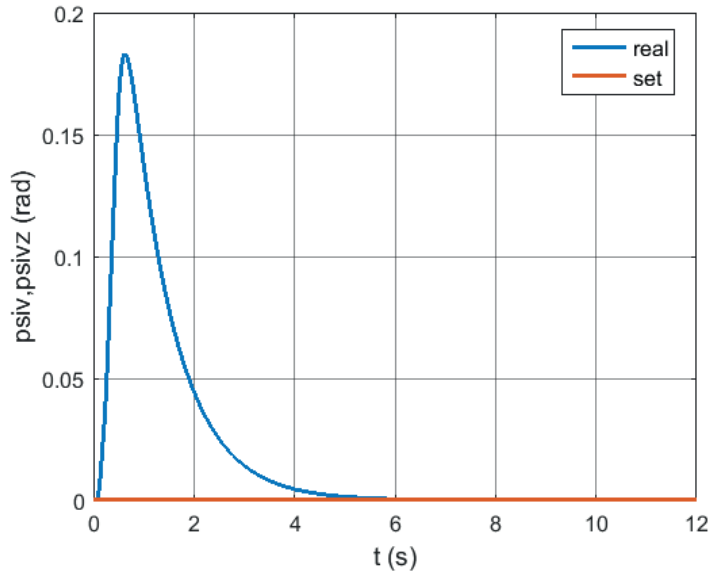

Figure 7 Changes of the state variables psiv and psivz - real and set - as a function of time

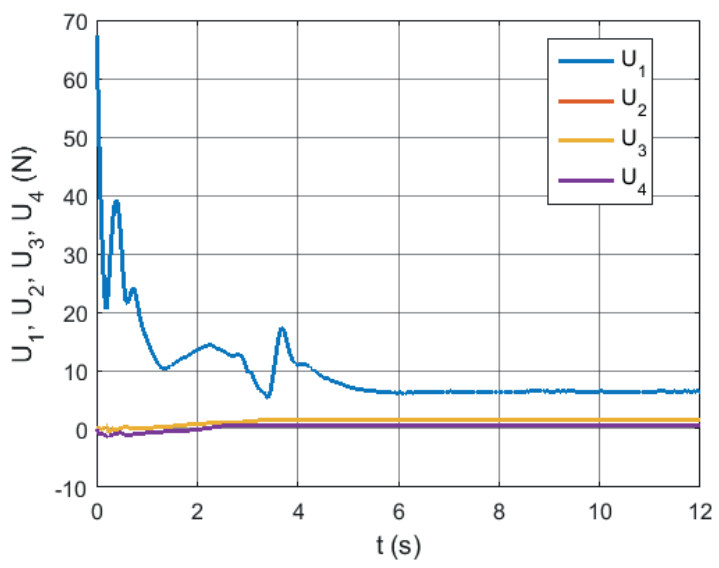

Figure 8 Changes of the drone's controls $U_{1}, U_{2}, U_{3}, U_{4}$ as a function of time

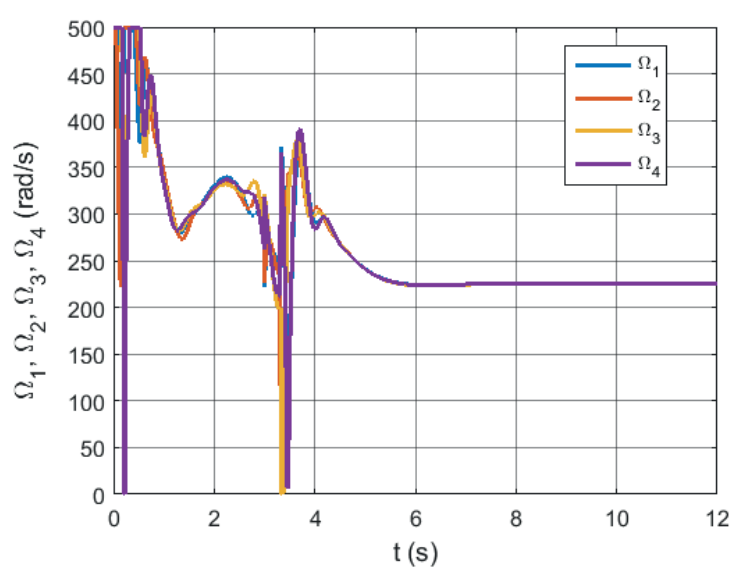

Figure 9 Changes of angular velocities of the drone's rotors as a function of time

impact on accuracy of the target illumination by the gyro axis. In figures, the position of the gyro axis determines the angles tetag and psig (blue), while the set position is tetagz and psigz angles (yellow) and angles determined by the observer are tetagobs and psigobs.

Initial simulations demonstrated that the system laser illuminating ground target from the deck of a drone,

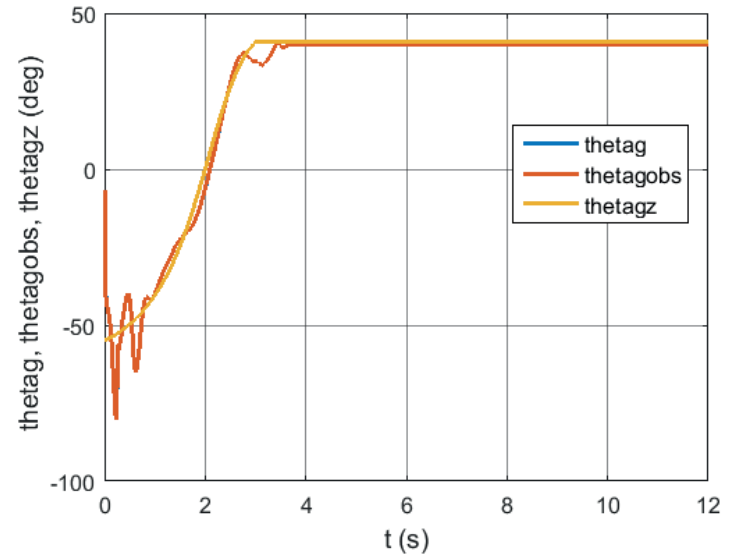

Figure 10 Changes of the state variables tetag, tetagobs and tetagz - real, observer and set - as a function of time

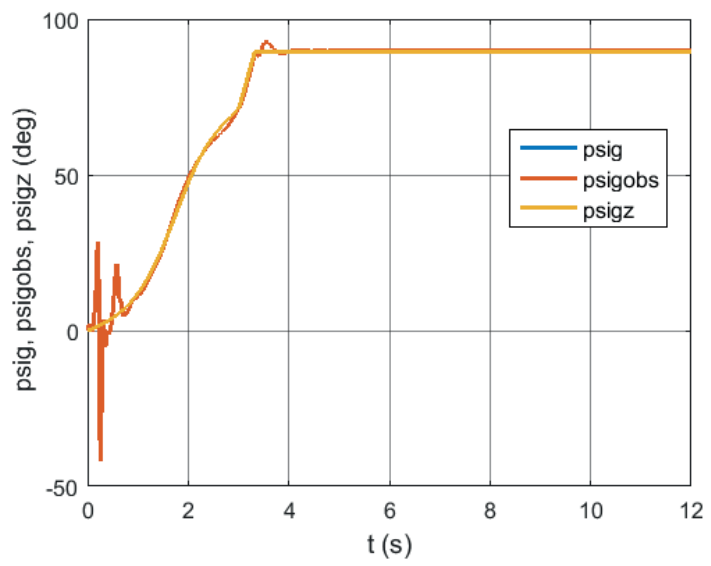

Figure 11 Changes of the state variables psig, psigobs and psigz - real, observer and set - as a function of time

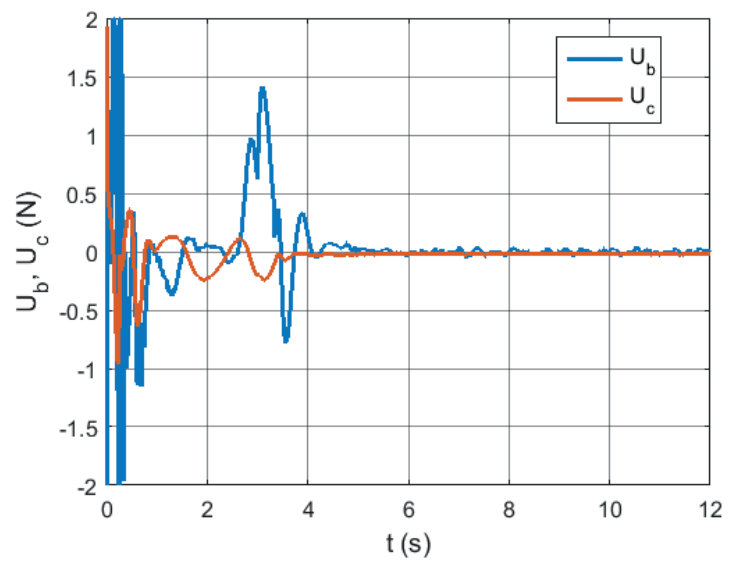

Figure 12 Changes of the gyro controls $U_{b}$ and $U_{c}$ as a function of time

using the gyro scanning and tracking head, is robust to large external interference from QCUAV and even its complete descent from both the pre-set trajectory and the hovering.

In addition, the gyro head and drone control algorithm proposed in this paper works correctly and ensures the precise laser target illumination capability. 


\section{References}

[1] KORUBA, Z., KRZYSZTOFIK, I. Nonlinear model of quadrotor dynamics during observation and laser target illumination. In: LACARBONARA, W., BALACHANDRAN, B., MA, J., TENREIRO MACHADO, J., STEPAN, G. (eds) Nonlinear dynamics and control. Springer, 2020, p. 187-196. ISBN 978-3-030-34746-8.

[2] KRZYSZTOFIK, I., KORUBA, Z. Analysis of quadcopter dynamics during programmed movement under external disturbance. In: LACARBONARA, W., BALACHANDRAN, B., MA, J., TENREIRO MACHADO, J., STEPAN, G. (eds) Nonlinear dynamics and control. Springer, 2020, p. 177-185. ISBN 978-3-030-34746-8.

[3] BOUABDALLAH, S. Design and control of quadrotors with application to autonomous flying. PhD thesis. EPFL, 2007.

[4] BRESCIANI, T. Modelling, identification and control of a quadrotor helicopter. Master Thesis. Lund University, 2008.

[5] NAIDOO, Y., STOPFORTH, R., BRIGHT, G. Quad-rotor unmanned aerial vehicle helicopter modelling and control. International Journal of Advanced Robotic Systems [online]. 2011, 8(4), p. 139-149. ISSN 1729-8814. Available from: https://doi.org/10.5772/45710

[6] KRZYSZTOFIK, I., KORUBA, Z. Mathematical model of movement of the observation and tracking head of an unmanned aerial vehicle performing ground target search and tracking. Journal of Applied Mathematics [online]. 2014, 2014, 934250. ISSN 1110-757X. Available from: https://doi.org/10.1155/2014/934250

[7] ZUO, Z. Quadrotor trajectory tracking control: a PD control algorithm. In: 3rd International Conference on Computer and Electrical Engineering: proceeding. Vol. 53(2). IACSIT Press, 2012. ISSN 2010-460X.

[8] MANJUNATH, A., MEHROK, P., SHARMA, R., RATNOO, A. Application of virtual target based guidance laws to path following of a quadrotor UAV. In: International Conference on Unmanned Aircraft Systems ICUAS 2016: proceedings. IEEE, 2016. ISBN 978-1-4673-9334-8, p. 252-260.

[9] BOUABDALLAH, S., SIEGWART, R. Backstepping and sliding-mode techniques applied to an indoor micro quadrotor. In: IEEE International Conference on Robotics and Automation: proceedings. IEEE, 2005. ISBN 0-7803-8914-X, p. $2259-2264$.

[10] ZULU, A., JOHN, S. A review of control algorithms for autonomous quadrotors. Open Journal of Applied Sciences [online]. 2014, 4(14), p. 547-556. ISSN 2165-3917. Available from: https://doi.org/10.4236/ojapps.2014.414053

[11] THU, K.M., GAVRILOV, A.I. Designing and modeling of quadcopter control system using L1 adaptive control. Procedia Computer Science [online]. 2017, 103, p. 528-535. ISSN 1877-0509. Available from: https://doi.org/10.1016/j. procs.2017.01.046

[12] BOUABDALLAH, S., NOTH, A., SIEGWART, R. PID vs LQ control techniques applied to an indoor micro quadrotor. In: IEEE/RSJ International Conference on Intelligent Robots and Systems IROS 2004: proceedings. IEEE, 2004. ISBN 0-7803-8463-6, p. 2451-2456.

[13] HE, Z., ZHAO, L. A Simple attitude control of quadrotor helicopter based on Ziegler-Nichols rules for tuning PD parameters. The Scientific World Journal [online]. 2014, 2014, 280180. ISSN 2356-6140. Available from: https://doi.org/10.1155/2014/280180

[14] KADA, B., GHAZZAWI, Y. Robust PID controller design for an UAV flight control system. In: World Congress on Engineering and Computer Science: proceedings. 2011. Vol. II. ISBN 978-988-19251-7-6.

[15] PRAVEEN, V., PILLAI, A. S. Modeling and simulation of quadcopter using PID controller. International Journal of Control Theory and Applications. 2016, 9(15), p. 7151-7158. ISSN 0974-5572.

[16] HASSENI, S., ABDOU, L. Decentralized PID control by using GA optimization applied to a quadrotor. Journal of Automation, Mobile Robotics and Intelligent Systems [online]. 2018, 12(2), p. 33-44. ISSN 1897-8649. Available from: https://doi.org/10.14313/JAMRIS_2-2018/9

[17] AWREJCEWICZ, J. KORUBA, Z. Classical mechanics: applied mechanics and mechatronics. New York: Springer, 2012. ISBN 978-1-4614-3978-3.

[18] KRZYSZTOFIK, I., KORUBA, Z. Application of the sliding controller for the gyroscope system of the anti-aircraft missile. In: 23rd International Conference Engineering Mechanics 2017: proceedings. Brno University of Technology, 2017. ISBN 978-80-214-5497-2, p. 530-533.

[19] KORUBA, Z., KRZYSZTOFIK, I. A control with the use of LQR modified method in the gyroscope system of target tracking. In: 23rd International Conference Engineering Mechanics 2017: proceedings. Brno University of Technology, 2017. ISBN 978-80-214-5497-2, p. 486-489.

[20] KORUBA, Z., KRZYSZTOFIK, I. Control of the non-stationary gyroscopic system in the target tracking process. In: 24th International Conference Engineering Mechanics 2018: proceedings. Institute of Theoretical and Applied Mechanics of the Czech Academy of Sciences, 2018. ISBN 978-80-86246-88-8, p. 397-400.

[21] TEWARI, A. Modern control design with Matlab and Simulink. Chichester: John Wiley \& Sons, 2002. ISBN 0-471-49679-0.

[22] LEWIS, F. L., VRABIE, D. L., SYRMOS, V. L. Optimal control. Hoboken: John Wiley \& Sons, 2012. ISBN 978-0-470-63349-6. 